Slavica

bruxellensia

\section{Slavica bruxellensia}

Revue polyphonique de littérature, culture et histoire

slaves

$11 \mid 2015$

Littérature et philosophie

\title{
Ben-Tovit de Leonid Andreev (1905) : un calvaire à la Nietzsche
}

\section{Serge Rolet}

\section{OpenEdition}

Journals

Édition électronique

URL : http://journals.openedition.org/slavica/1699

DOI : 10.4000/slavica.1699

ISSN : 2034-6395

\section{Éditeur}

Université libre de Bruxelles - ULB

\section{Référence électronique}

Serge Rolet, «Ben-Tovit de Leonid Andreev (1905) : un calvaire à la Nietzsche », Slavica bruxellensia [En ligne], 11 | 2015, mis en ligne le 25 février 2015, consulté le 19 avril 2019. URL : http:// journals.openedition.org/slavica/1699; DOI : 10.4000/slavica.1699

Ce document a été généré automatiquement le 19 avril 2019

\section{(c) $(1) \odot$}

Les contenus de Slavica bruxellensia sont mis à disposition selon les termes de la Licence Creative Commons Attribution - Pas d'Utilisation Commerciale - Pas de Modification 3.0 France. 


\title{
Ben-Tovit de Leonid Andreev (1905): un calvaire à la Nietzsche
}

\author{
Serge Rolet
}

\section{Article}

\section{Introduction}

1 Dans la culture russe des années 1890-1910, la référence, explicite ou non, à Friedrich Nietzsche est omniprésente. En Russie comme ailleurs en Europe à cette époque, les courants intellectuels, artistiques et littéraires les plus divers sont requis par la parole du philosophe. À la faveur de traductions précoces et de commentaires remarqués, sa pensée donne lieu en Russie à une floraison de thèmes (le renversement des valeurs), de figures (le surhomme), de postures (une véhémence agressive, un ton prophétique, etc.)‥ La «combinabilité» de Nietzsche avec la culture russe est étonnamment élevée, et l'inventaire raisonné de ses produits est encore aujourd'hui un défi à la recherche². Néanmoins, certaines grandes tendances apparaissent. On voit se dessiner «trois Nietzsche russes", le premier symboliste, le second marxiste, et le troisième existentialiste ${ }^{3}$.

2 On s'arrêtera ici sur Leonid Andreev (1871-1919). Très sensible à l'air du temps, habile à proposer un traitement inattendu et volontiers provocateur des «questions éternelles ", cet auteur de nouvelles, qui, dans les premières années $d u x^{e}$ siècle, fait partie de l'entourage de Maksim Gor'kij, lit Nietzsche dans une perspective révolutionnaire. Le récit Ben-Tovit, à l'exemple de la pensée de l'éternel retour chez Nietzsche, peut être compris de deux manières différentes, l'une classique, à la portée de tous (Ben-Tovit s'oppose à Jésus), l'autre, bien plus difficile, et requérant de la part du lecteur une capacité àsupporter « la pensée la plus lourde » (Ben-Tovit et Jésus sont semblables). 


\section{Les échos à Nietzsche dans les récits d'Andreev}

3 Le héros de Rasskaz o Sergee Petroviče (L'Histoire de Sergej Petrovič, 1900), l'une des premières œuvres d'Andreev, est un pauvre étudiant, terne et un peu lent d'esprit, qui s'adonne à la lecture d'Also sprach Zarathustra (Ainsi parlait Zarathoustra) ${ }^{4}$. Il est comparé à un cheval de trait, et quand il se révolte, c'est « comme un esclave ». Sergej Petrovič est la énième version $\mathrm{du}$ "petit homme", personnage emblématique de la littérature émancipatrice russe depuis GogoL'. En termes nietzschéens, il correspond au stade du chameau. Le seul précepte nietzschéen qui soit à sa portée est le suivant : « Il en est qui manquent leur vie; un ver venimeux leur ronge le cœur. Qu'ils tâchent au moins de réussir d'autant mieux leur mort. $»^{5}$ Alors, Sergej Petrovič se suicide. Mais sa mort n'a rien de très « réussi » : le poison qui doit le tuer est mal dosé, son agonie est interminable, pitoyable, minable. À vouloir jouer au surhomme, croit-on comprendre, le lecteur lambda de Nietzsche risque le ridicule. Il ne faut pas singer Nietzsche, comme le font les symbolistes. Comment ne pas parodier Nietzsche, comment s'imprégner de sa pensée sans devenir, pour autant, grotesque?

Quelques années après Rasskaz o Sergee Petroviče, Andreev publie Ben-Tovit (1905), un très court récit, très peu commenté, que le célèbre critique Kornej Čukovskij trouvait des plus réussis ${ }^{6}$. Dans cette anecdote, dont le lecteur ne sait pas s'il doit la trouver énigmatique ou simplement insignifiante, on ne relève aucune allusion, aucune référence, même indirecte, à Nietzsche. Ici, pas de surhomme, pas de chameau, pas de belle brute blonde, pas de prédication dans le ton de Zarathoustra. Pourtant, ce texte a bel et bien quelque chose de nietzschéen dans sa démarche : il ne contient pas à proprement parler de thèse, que le lecteur aurait simplement à comprendre, mais, ce qui est très différent, il fait appel à sa capacité de décider. Percevoir Ben-Tovit comme un écho à Nietzsche permet de sortir de l'énigme, et d'éviter une interprétation standard.

\section{La lecture standard du récit (Jésus vs. Ben-Tovit) et ses insuffisances}

5 La lecture de Ben-Tovit qui vient automatiquement à l'esprit est à peu près la suivante : parmi nous, hélas, il y a des gens dont l'univers est tellement limité, qui sont tellement incapables de se soucier d'autre chose que de leurs petits intérêts matériels, que, même s'ils assistaient à la Passion du Christ, une simple rage de dents suffirait à les empêcher d'y accorder la moindre attention.

6 Regardez, semble suggérer Andreev, comment l'horizon de ce boutiquier, un brave type, comme son nom l'indique, est étroit : il ne pense qu'au jeune âne qu'il vient d'acheter, une bonne affaire. «L'injustice du monde » ne le concerne pas. Le récit est allégorique. Le marchand de Jérusalem remonte à Belikov, le personnage de Čelovek $v$ futljare (L'Homme à l'étui) d'Anton Čekhov, mais il est aussi une figure du meščanin (« petit-bourgeois »), cible principale des écrivains de la pléiade gorkienne ${ }^{7}$. Ce que le petit-bourgeois mesquin est incapable de voir, c'est la révolution qui approche, comme l'orage : en ce début du $\mathrm{xx}^{\mathrm{e}}$ siècle, on l'entend déjà gronder. Le texte a une fonction critique. Le lecteur ne peut que se désolidariser de la médiocrité du boutiquier de Jérusalem, et aspirer aux «lointains obscurs » de la révolution'. Cette lecture, qui donne à Ben-Tovit à peu près le même sens qu'à Nevesta (La Fiancée) de Čekhov, met en relief l'opposition, habituelle dans le récit 
court, entre les deux pôles du texte: Ben-Tovit s'oppose à Jésus comme ce qui est petit s'oppose à ce qui est grand'.

7 L'interprétation standard de Ben-Tovit n'est pas très satisfaisante, pour deux raisons. D'abord, un certain nombre d'indices empêchent d'opposer Jésus et Ben-Tovit aussi simplement qu'on vient de le faire. Plusieurs éléments établissent au contraire dans le texte un parallélisme entre les deux personnages. La souffrance est éprouvée par eux au même endroit, au même moment. Nous savons que la flagellation du Christ a lieu la veille de sa crucifixion (Luc, 22 : 62-66). Dans Ben-Tovit, nous trouvons :

Le jour terrible où s'accomplit l'injustice du monde et où Jésus-Christ fut crucifié sur le Golgotha, au milieu des bandits, ce jour-là le négociant de Jérusalem BenTovit fut pris dès le matin d'une rage de dents insupportable. Cela avait commencé la veille au soir.

Le rapprochement que nous sommes amenés à établir entre Jésus et Ben-Tovit repose autant sur des éléments internes au texte (" le jour terrible », " ce jour-là »), que sur un parallélisme entre eux et des éléments externes, mais forcément présents à la mémoire du lecteur de l'époque. Le lendemain du "jour terrible», Ben-Tovit ne ressent plus de douleur. Là où sa joue était toute gonflée, il n'y a presque plus rien. Samuel constate que la trace visible de ses tourments a disparu, tout comme, dans l'Évangile, on découvre que le tombeau de Jésus est vide. L'analogie est vague, un peu bancale, mais pour être bancale, elle n'en est pas moins fonctionnelle : l'analogie est de la responsabilité du lecteur, c'est lui qui la fait ressortir, sur le fond d'indices à moitié concluants, et tant pis s'il ne s'avise pas de la remarquer.

8 La Passion du Christ et la rage de dents du boutiquer coïncident. Une série de détails, qui passent facilement inaperçus à une lecture rapide, confirment l'analogie entre les souffrances de Jésus et celles de Ben-Tovit. Les « milliers de clous chauffés au rouge » et «l'aiguille brûlante » auxquels est comparée la douleur de Ben-Tovit, font signe vers les clous de la mise en croix.

(...) la bouche et la tête de Ben-Tovit étaient toutes pleines d'une horrible sensation de souffrance, comme si on l'avait forcé à mâcher des milliers de clous chauffés au rouge.

Les cris redoublèrent et la foule, telle une vague marine multicolore, submergea celui qui s'était écroulé. Ben-Tovit tressaillit brusquement de douleur - c'était comme si quelqu'un lui avait enfoncé dans la dent une aiguille brûlante (...).

Un peu plus haut, il est dit que les dents de Ben-Tovit «ondulèrent comme une vague ». Certes, un grand nombre de détails précisant le comportement de Ben-Tovit suggèrent qu'il n'a pas grand-chose de commun avec Jésus, bien qu'il soit un brave homme: il s'emporte, se montre autoritaire, et prosaïque à l'excès. Mais ces traits, qui l'opposent à Jésus, donnent d'autant plus de relief à un point particulier. Deux expériences différentes de la douleur physique, faites par des personnes que tout oppose, sont semblables, et sont quelquefois dites avec les mêmes mots. Au lieu de faire des deux figures majeures du récit deux pôles entre lesquels passe une tension électrique, comme la structure de la nouvelle classique le montre habituellement, Andreev construit au fil du texte une équivalence entre elles. Plus tard, dans le récit Iuda Iskariot (Judas Iscariote, 1907), comme dans le pastel qu'il en a tiré plus tard, intitulé Cari Iudejskie (Les Tsars de Judée), où l'on voit le Christ et Judas côte à côte, Andreev reprend ce procédé : les deux figures antithétiques sont équivalentes, à la polarité traditionnelle se substitue une similitude impensable, choquante ${ }^{10}$. 


\section{L'œuvre révolutionnaire doit être irrécupérable}

10 Andreev était convaincu que la représentation littéraire, fût-elle critique, était politiquement inefficace. La prose de l'époque a en effet, à ses yeux, le défaut de déclencher une expérience esthétique, plutôt que de pousser le lecteur à agir. Le public, amateur d'émotion artistique, peut «récupérer» ce que la vérité de la littérature a d'insupportable, et l'intégrer à son mode de vie confortable. La charge politique est neutralisée par la beauté du texte. Andreev partage le rejet de la représentation littéraire exprimé par Vikentij Veresaev (1867-1945). Celui-ci avait publié un peu plus tôt un récit dans lequel un écrivain répondait en ces termes aux louanges que lui adressait le public, dans l'une des soirées littéraires qui faisaient fureur à l'époque, à Moscou, SaintPétersbourg, et dans les lieux de villégiature de Crimée :

Oui, la force de l'art est grande, mais ce n'est pas sa capacité à éveiller les «bons sentiments » qui est grande. La force, maudite et corruptrice, de l'art réside en ce qu'il dénature et estropie de la manière la plus incroyable tout sentiment, tout mouvement que la réalité fait naître dans l'âme. L'artiste est prêt à fustiger la vie, mais au moment où il frappe, son fouet se transforme en une douce guirlande de muguet parfumé. (...) Les artistes, à commencer par Tolstoj, Hugo, Dostojevskij et pour finir avec nous, les petits, vous ont fait éprouver, sans peine ni douleur, les plus pénibles des catastrophes intérieures. Et vous en êtes repus. Vous êtes las de lutter sans avoir lutté, las d'aimer sans avoir aimé.

(...) On l'a dit depuis longtemps : «C'est par la parole que l'écrivain agit ». C'est bien possible. Mais ce qui est vrai, c'est que cette action reste simplement verbale, et en nos cœurs nous comprenons très bien ce que cette parole-action a de monstrueusement artificiel. ${ }^{11}$

11 Pour sa part, Andreev avait pris le parti de la provocation, censée empêcher le confort démobilisateur, et donner à la littérature sa pleine efficacité performative. Dans le récit «Bezdna » (Le Gouffre, 1902), il avait soulevé un énorme scandale, et d'interminables débats dans la presse. Lev Tolstoj lui-même avait exprimé son émotion. Andreev, lui, se délectait d'avoir perturbé le lecteur, l'empêchant ainsi de jouir du texte, et reprenait ce distique, comme un refrain :

Bud'te ljubezny Que quelqu'un lise "Le Gouffre»

Ne čitajte "Bezdny" Cela, on ne le souffre. ${ }^{12}$

Brouillant la limite entre art littéraire (khudožestvennaja literatura) et littérature de circonstance (publicistika), Andreev affirme écrire des récits " non artistiques », justifiés par la seule urgence politique. Dans le cercle littéraire «Sreda ( (Mercredi), auquel il appartenait, la vocation émancipatrice de la littérature n'était pas censée remettre en cause la supériorité traditionnelle de l'art littéraire sur la littérature de circonstance. La position d'Andreev était perçue comme une forme de surenchère.

12 La révolution est certes dirigée avant tout contre l'ordre politique et social de Nicolas II, mais cette orientation est bien générale : le pouvoir est loin. Au sein du champ littéraire russe de l'époque, l'ennemi majeur de la révolution n'est autre que la petite-bourgeoisie ( meščanstvo $)^{13}$.

Dans l'ambiance particulière de la période qui précède les événements révolutionnaires de 1905, on voit apparaître chez Andreev, une tentative visant à faire tomber les masques ${ }^{14}$. Parmi toutes les forces qui se soulèvent contre l'autocratie et le gouvernement de l'Empire, il faut séparer les révolutionnaires véritables des faux, de ceux qui adoptent seulement la posture de l'opposition, valorisante dans les milieux intellectuels et assez 
peu risquée. Il faut éprouver jusqu'où vont les convictions de chacun, établir les limites de ce que les intellectuels sont prêts à renverser, de ce à quoi ils sont capables de renoncer. Symboliquement, la révolution est ce qui abat les barrières. Symétriquement, sera dit "petit-bourgeois» celui qui, selon la formule gorkienne, "aime élever partout des barrières » (ljubit vezde vozdvigaŤ ogrady) ${ }^{15}$. « Le pauvre petit-bourgeois peut bien avancer, car ça rapporte, si ce n'est pas risqué, et c'est agréable, si ça ne coûte pas cher. Mais aller plus haut, il en est incapable. Il pèse trop lourd, ce fils de chien! Il pèse trop lourd ! Il s'est empiffré de culture (kuL'turoj on obožralsja). $»^{16}$

CEuvrer en faveur de la révolution, c'est avant tout démasquer le petit-bourgeois qui se cache sous l'intellectuel contestataire, à une époque où, de toute manière, il est difficile de trouver dans ce milieu quelqu'un qui soutienne le gouvernement. Un texte comme «Bezdna ", déjà, faisait office de test: ceux qui étaient capables de supporter le récit étaient du bon côté, non les autres.

Pour Andreev, écrivain alternatif, le public (le lecteur-type, le spectateur de théâtre) se confond avec l'intellectuel et, donc, aussi bien, avec le petit-bourgeois À l'époque de BenTovit, le nec plus ultra est de rabrouer le public, de l'agresser, et de refuser la «gloire». Voici l'une des nombreuses mises en garde que Gor'kij adresse à Andreev :

J'ai grande envie qu'on te haïsse, et même à l'extrême, car, vois-tu, chaque fois que le nez de mon âme sent l'odeur de la haine, je gagne en intelligence et en talent, ce dont tu as besoin, toi aussi. Toi, même davantage que moi. Car la gloire, j'en ai assez reniflé l'odeur (elle sent l'aigre, la gloire, en Russie !), tandis que toi, pas encore. Crains les louanges plus que tout, oh, Leonid! Chaque fois que j'en entends, j'ai l'impression que ça vient d'un fils de chien, et qu'il fait mon éloge parce qu'il a peur, le pauvre, que je lui dise ses quatre vérités. Elles sont douteuses, les louanges du petit-bourgeois, habitué à graisser la patte à tout le monde, du planton jusqu'au bon Dieu. ${ }^{17}$

On voit fonctionner ici dans des enjeux nouveaux la vieille dichotomie, posée par Mikhail Saltykov-Ščedrin (1826-1889), du «lecteur-ami» et du «lecteur-ennemi ». Apparaît comme ami le lecteur capable d'approuver la provocation, autrement dit de saisir et d'assumer pour son propre compte la dimension performative du texte. Le lecteurennemi est celui qui condamne les textes "révolutionnaires ", dans lesquels il perçoit une mise en cause des fondements de la civilisation, une transgression des grands interdits, et ainsi de suite.

La lecture conventionnelle de Ben-Tovit, celle qui oppose Ben-Tovit à Jésus, est en retrait par rapport à la conception qu'Andreev se faisait de la littérature. Elle fait du texte « une guirlande de muguet parfumé ", alors qu'il faut " cogner » le petit-bourgeois esthète ${ }^{18}$. Pour percevoir la charge "révolutionnaire", déstabilisatrice, du récit, il faut revenir à l'analogie entre les deux pôles, entre Jésus et le petit boutiquier de Jérusalem.

\section{L'interprétation comme décision : une épreuve à surmonter}

L'interprétation standard du récit, qui occulte le parallélisme entre Jésus et le boutiquier, peut être rapprochée, chez Nietzsche, du refus de l'éternel retour. Mais le fait de percevoir ce parallélisme ne garantit pas encore une bonne lecture du texte, de la même manière que, dans Zarathoustra, formuler la thèse de l'éternel retour ne suffit pas à sortir du nihilisme. Chez Andreev comme chez Nietzsche, il faut quelque chose de plus, qui n'engage plus seulement l'entendement, mais la volonté. Des deux interprétations qui s'offrent au lecteur, la plus féconde est la plus difficile à supporter. 
18 Au début de la troisième partie du Zarathoustra, au chapitre « Vom Gesicht und Rätsel » (La vision et l'enigme), la pensée de l'éternel retour est évoquée comme une dualité. Zarathoustra conte son dialogue avec un nain qu'il a longtemps porté sur son dos, sur un sentier de montagne :

Regarde cette poterne, gnome, lui dis-je encore. Elle a deux issues. Deux chemins se rejoignent ici ; nul ne les a suivis jusqu'au bout.

Cette longue route qui s'allonge derrière nous dure une éternité. Et cette longue route qui s'étire devant nous, c'est une autre éternité.

Ces chemins se contrecarrent; ils se heurtent du front, et c'est ici, sous cette poterne, qu'ils se rencontrent. Le nom de la poterne est inscrit au fronton ce nom, c'est l'Instant.

Mais si quelqu'un suivait l'une de ces routes, sans arrêt et jusqu'au bout, crois-tu, gnome, que ces routes s'opposeraient toujours ? ${ }^{19}$

Cette question reçoit successivement deux réponses. Celle du nain est la suivante : - Tout ce qui est droit est menteur, murmura le nain d'un ton méprisant. Toute vérité est courbe, le temps lui-même est un cercle. ${ }^{20}$

La vision du nain est celle de "l'Esprit de Pesanteur». Elle provoque la colère de Zarathoustra. Une autre manière de concevoir l'éternel retour est possible :

Et toutes choses ne sont-elles pas si solidement enchevêtrées que cet instant présent entraîne à sa suite toutes les choses futures ? Et lui-même par conséquent? 21

Le nain, figure du nihilisme, choisit de faire de l'Instant la répétition de ce qui a déjà été. L'Instant advient à la fin du chemin, quand l'éternité est déjà « derrière ». Zarathoustra, au contraire, le voit aussi comme ce qui marque l'avenir pour toujours : l'éternité est encore « devant », et la volonté peut décider de son cours.

La pensée de l'éternel retour, "ma pensée d'abîme ", dit Zarathoustra, est difficile à supporter. Elle est ce que Zarathoustra «portai[t] de meilleur et de pire $»^{22}$. Le récit du dialogue avec le nain est immédiatement suivi (dans le même chapitre) d'une autre vision, dans laquelle, incité par Zarathoustra, un jeune pâtre se libère du serpent qui lui est entré dans la gorge, en lui tranchant la tête de ses dents. Le serpent dont il faut couper la tête figure le dégoût de l'éternel retour : si tout doit revenir, alors le nihilisme reviendra lui aussi éternellement. Il faut surmonter ce dégoût.

De Nietzsche à Andreev, la thématique change (de l'éternel retour, on passe à la Passion du Christ), et une grande partie du fonctionnement même de l'énigme. Le statut de ce qu'il faut supporter n'est pas le même. Dans le Zarathoustra, le dégoût de l'éternel retour est provoqué par la présence de fait du nihilisme, qui n'a en lui-même rien de précieux. Dans Ben-Tovit, au contraire, la supériorité de l'une des deux interprétations du texte découle essentiellement de ce qu'elle contient d'insupportable. L'effet prime sur le contenu. L'idée que le christianisme est un mythe parmi d'autres (Jésus est simplement un homme, il a souffert comme Ben-Tovit, il est à l'image de l'homme, comme dans toute religion, ou à peu près) n'a guère d'intérêt pour Andreev, elle est juste un rouage dans un jeu pragmatique, seul compte le résultat qu'elle produit, sa capacité à faire tomber les masques. De même, le récit d'Andreev ne présente pas expressément, comme on le voit chez Nietzsche, deux interprétations successives, et opposées, de la même histoire. À vrai dire, il n'en livre aucune. Mais le caractère stéréotypé du décryptage le plus immédiatement évident laisse supposer qu'il en existe un autre. La structure duale de Ben-Tovit est hypothétique.

21 Ces différences sont très profondes, mais elles ne tendent pas jusqu'à le rompre le fil qui relie Ben-Tovit à «La vision et l'enigme ». Même s'il ne faut pas considérer Andreev 
comme un disciple conséquent de Nietzsche, on peut admettre que, dans Ben-Tovit, la construction du sujet est bel et bien nietzschéenne. De la même manière que dans « La vision et l'énigme ", ce qui est en jeu n'est pas simplement une thèse, un énoncé auquel on attribue, ou non, une valeur de vérité, mais la capacité à en supporter une interprétation particulière. Dans la pensée de l'éternel retour, "l'Esprit de Pesanteur » voit que le temps est un cercle, tout comme le petit-bourgeois «trop lourd» comprend que la Passion du Christ et la rage de dents d'un marchand de Jérusalem sont semblables, mais ni l'un, ni l'autre ne peuvent s'élever à une interprétation supérieure.

Zarathoustra surmonte le dégoût de l'éternel retour; Andreev met le lecteur de Ben-Tovit au défi de supporter l'idée que, peut-être, c'est Jésus qui souffre comme le boutiquier de Jérusalem, et non l'inverse. Si l'on veut ébranler le monde, il faut s'attaquer à ses fondements, détruire les repères ultimes, porter l'angoisse dans l'éthos étriqué du petitbourgeois, trop attaché à la stabilité des choses. La bonne manière de comprendre le récit dépend d'une décision infiniment difficile, et c'est bien là ce qui fait de lui un test, révélant si le lecteur est aussi révolutionnaire qu'il le prétend. Tout le monde, ou presque, croit l'être, et Ben-Tovit est un récit " pour tous ", mais en fin de compte, peu nombreux sont ceux qui sont capables de bien le lire, et Ben-Tovit est un récit " pour personne ».

Ben-Tovit donne l'occasion de sortir du vague qui entoure l'idée générale, et probablement justifiée, selon laquelle Andreev s'inspire de Nietzsche. La relation entre les deux auteurs n'est en effet explicite que dans Rasskaz o Sergee Petroviče. Il est possible de déchiffrer BenTovit comme une transposition libre, et peut-être largement inconsciente, de «La vision et l'enigme ». La référence à Nietzsche peut se justifier génétiquement par le fait qu'Andreev s'était longuement imprégné du Zarathoustra, et par la convergence, par-delà toutes les différences de convictions entre les deux auteurs, des traits qui caractérisent l'ennemi : le " petit-bourgeois » appartient globalement au même topos que "l'Esprit de Pesanteur ». Mais tout le monde, à cette époque, lisait et relisait le Zarathoustra, et Andreev n'est pas le seul à s'en prendre au petit-bourgeois. On découvre dans Ben-Tovit que la proximité particulière de notre auteur avec Nietzsche repose sur une certaine convergence de leur relation avec les idées. Pour lui comme pour le philosophe, les idées ne se réduisent pas à leur contenu purement thétique, leur sens dépend essentiellement d'un choix de la volonté. Il semble bien que, sur ce point précis, Andreev soit plus proche de Nietzsche qu'aucun des écrivains russes du début du xxe siècle.

\section{Annexe : traduction de Ben-Tovit}

Le jour terrible où s'accomplit l'injustice du monde et où Jésus-Christ fut crucifié sur le Golgotha, au milieu des bandits, ce jour-là le négociant de Jérusalem Ben-Tovit fut pris dès le matin d'une rage de dents insupportable. Cela avait commencé la veille au soir : le côté droit de la mâchoire s'était mis à lui lancer et c'était comme si une dent, la dernière avant la dent de sagesse, était un peu plus haute, et quand la langue passait dessus, elle lui faisait un peu mal. Après le repas, pourtant, la douleur s'était calmée tout à fait, et, rasséréné, Ben-Tovit l'avait oubliée : il avait le jour même échangé son vieil âne contre une bête jeune et robuste, il était tout guilleret et n'avait pas prêté sens à ces mauvais présages.

Et il avait très bien et très profondément dormi, mais avant le lever du jour, quelque chose avait commencé à l'alerter, comme si on l'appelait pour une affaire de première importance, et quand Ben-Tovit se réveilla, de méchante humeur, il avait une rage de 
dents, une rage de dents franche et mauvaise, qui le tenaillait de manière aiguë et intense. Et il n'était déjà plus question de savoir si c'était la même dent que la veille qui faisait mal, ou si la douleur venait aussi des dents voisines : la bouche et la tête de BenTovit étaient toutes pleines d'une horrible sensation de souffrance, comme si on le forçait à mâcher des milliers de clous chauffés au rouge. Il se bassina la bouche avec l'eau d'une cruche en terre ; le feu de la douleur disparut une minute, ses dents furent prises d'une convulsion et ondulèrent comme une vague, et cette sensation fut même agréable, comparée à la précédente. Ben-Tovit se recoucha, se rappela son nouvel âne, pensa combien il serait heureux si ses dents ne le tourmentaient pas, et chercha à se rendormir. Mais l'eau tiédit et, cinq minutes plus tard, la douleur revint encore plus furieuse qu'avant, et Ben-Tovit, assis sur sa couche, se balançait comme le pendule d'une horloge. Son visage était tout ridé et se plissait autour de son grand nez, blême de douleur, au bout duquel perlait une gouttelette de sueur froide. C'est ainsi que, se balançant et gémissant, il reçut les premiers rayons du soleil, auquel il allait être donné de voir le Golgotha avec ses trois croix, et de pâlir d'horreur et d'amertume.

Ben-Tovit était un bon et brave homme qui haïssait l'injustice, mais quand sa femme se réveilla, il eut des mots désagréables et, n'ouvrant qu'à peine la bouche, il se plaignit qu'on l'eut laissé seul, comme un chien du désert, hurler et se tordre de douleur. Sa femme prit ces reproches immérités avec patience, car elle savait qu'ils n'avaient pas été dits par méchanceté, et apporta force remèdes : de la fiente de rat séchée à appliquer sur la joue, de la liqueur de scorpion et un petit morceau de roche, véritable éclat des tables de la Loi brisées par Moïse. La fiente de rat l'apaisa quelque peu, mais cela ne dura pas, ce fut la même chose avec la liqueur de scorpion et la pierre, mais à chaque fois, après une amélioration momentanée, la douleur revenait avec une force nouvelle. Et en ces courts instants de répit, Ben-Tovit se consolait en pensant à son âne et rêvait de lui, mais quand le mal empirait, il geignait, se mettait en colère contre sa femme et menaçait de se casser la tête contre une pierre, si la douleur ne se calmait pas. Et il allait sans cesse d'un coin à l'autre du toit en terrasse de sa maison, n'osant pas s'approcher du côté qui donnait sur la rue, car il avait la tête tout enrubannée dans un foulard, comme une femme. À plusieurs reprises ses enfants vinrent le voir pour lui raconter quelque chose d'une voix pressée à propos de Jésus de Nazareth. Ben-Tovit s'arrêtait, les écoutait une minute, le visage creusé de rides, mais bientôt il tapait du pied avec colère et les chassait: c'était un homme plein de bonté qui aimait ses enfants, mais maintenant il se fâchait qu'on l'importune pour trois fois rien.

Il était tout aussi désagréable de voir la rue et les toits avoisinants remplis de badauds qui ne faisaient rien que regarder avec étonnement Ben-Tovit et sa tête bandée comme celle d'une femme. Il s'apprêtait déjà à descendre quand sa femme lui dit : «Regarde, les voici qui amènent les bandits. Cela te distraira peut-être.

- Laisse-moi, s'il te plaît. Ne vois-tu pas comme je souffre ?» répondit Ben-Tovit avec colère.

Mais les paroles de sa femme sonnaient comme la vague promesse que sa rage de dents pouvait passer, et il s'approcha malgré lui du parapet. La tête penchée sur le côté, fermant un œil, la joue dans la main, il prit un visage dégoûté et larmoyant et regarda en bas.

Dans la rue étroite qui menait à la colline, une foule énorme avançait en désordre, entourée de poussière et de cris sans fin. Au milieu, ployant sous le poids des croix, avançaient les criminels, et au-dessus d'eux les fouets des soldats romains se tordaient comme des serpents. L'un d'eux, celui qui avait les cheveux clairs et la tunique déchirée et 
ensanglantée, heurta la pierre qu'on lui lançait dans les jambes et tomba. Les cris redoublèrent et la foule, telle une vague marine multicolore, submergea celui qui s'était écroulé. Ben-Tovit tressaillit brusquement de douleur - c'était comme si quelqu'un lui avait enfoncé dans la dent une aiguille brûlante et l'y avait remuée, il gémit : «ou-ouou », - et s'écarta du parapet, plein d'indifférence dégoûtée et d'irritation.

«Comme ils crient! » dit-il avec envie, en imaginant les bouches largement ouvertes aux dents fortes et saines et les cris qu'il aurait poussés lui-même, s'il avait été valide.

Et à cette idée, la douleur redoubla de fureur, et il secoua la tête à plusieurs reprises et beugla : «M-ou-ou...»

«On raconte qu'Il guérit les aveugles » dit sa femme, qui ne quittait pas le parapet, et elle jeta un caillou à l'endroit où, lentement, Jésus avançait, relevé par le fouet. « Mais bien sûr! Il n'a qu'à guérir la rage de dents que voici » ironisa Ben-Tovit et, irrité, il ajouta : "Qu'est ce qu'ils font comme poussière! Un vrai troupeau! Il faudrait les chasser tous à coups de bâtons ! Conduis-moi en bas, Sarah!»

Sa femme avait raison : le spectacle dérida un peu Ben-Tovit ; la fiente de rat avait peutêtre fini par agir, et il parvint à s'endormir. Et quand il se réveilla, la douleur avait presque disparu, et c'était tout juste si une légère fluxion lui gonflait le côté droit de la mâchoire, mais elle était si petite qu'on la remarquait à peine. Sa femme disait que cela ne se voyait pas du tout, mais Ben-Tovit souriait d'un air malicieux : il savait combien sa femme était bonne et aimait faire plaisir. Un voisin, le tanneur Samuel, arriva, et BenTovit l'emmena voir son âne et écouta avec orgueil les chaudes louanges que Samuel leur faisait, à lui-même et à l'animal.

Puis, à la demande de la curieuse Sarah, tous trois s'en allèrent au Golgotha voir les crucifiés. En chemin, Ben-Tovit raconta par le menu à Samuel comment, la veille, il avait ressenti une gêne dans la mâchoire, du côté droit, et comment, la nuit suivante, il avait été réveillé par une horrible douleur. Pour faire plus d'effet, il prenait un visage de martyr, fermait les yeux, secouait la tête et gémissait, tandis que Samuel, avec sa barbe blanche, opinait et disait : « Aïe-aïe-aïe ! Quelle douleur ! »

L'approbation plut à Ben-Tovit, et il répéta son récit, et ensuite il en vint aux temps éloignés où pour la première fois il avait eu une dent gâtée, en bas, à gauche. Conversant ainsi avec animation, ils arrivèrent au Golgotha. Le soleil, condamné à éclairer le monde en ce terrible jour, était descendu derrière les collines lointaines, et à l'ouest une traînée pourpre, comme une trace de sang, flambait. Les ombres indistinctes des croix s'y détachaient, et au pied de celle du milieu, des silhouettes agenouillées faisaient de vagues taches blanches.

Les gens étaient partis depuis longtemps : le froid tombait, et, après avoir jeté un regard furtif aux crucifiés, Ben-Tovit prit le bras de Samuel et le ramena avec précaution vers la maison. Il se sentait particulièrement en verve, et il avait envie d'achever son histoire de rage de dents. Ils marchèrent donc, et sous les signes de compassion et les exclamations de Samuel, Ben-Tovit faisait une mine de martyr, secouait la tête et gémissait avec art, alors que la nuit noire montait des gorges abruptes, des plaines lointaines et calcinées. Comme si elle avait voulu cacher aux regards du ciel le suprême méfait de la terre. 


\section{NOTES}

1. Le livre du germaniste français Henri Lichtenberger, La Philosophie de Nietzsche (1898) est paru en russe sous la direction de Mikhail Nevedomskij (pseud. de Miklaševskij), izd. Popovoj, SaintPétersbourg, 1900 ; voir également Šestov L., Dobro v učenii Dostoevskogo i Nitcše (Le Bien dans la doctrine de Dostoevskij et de Nietzsche), tipogr. Mikhaila Stasuleviča, Saint-Pétersbourg, 1900.

2. Parmi la très riche bibliographie, signalons simplement le recueil d'articles Nietzsche in Russia (Nietzsche en Russie), sous la direction Rosenthal B. G., Princeton University Press, Princeton, 1986.

3. Cf. Aucouturier M., "Les trois Nietzsche russes ", in: Le Rayonnement européen de Nietzsche, sous la direction de Merlio G. \& D'Iorio P., Klincksieck, Paris, 2004, pp. 89-99.

4. Andreev L., Rasskaz o Sergee Petroviče (L'Histoire de Sergej Petrovič), in : Žizn', t. X, 1900, rééd. in : Sobr. soč. v 6-i tomakh (đuvres en six volumes), t. I, Hudožestvennaja literatura, Moscou, 1990, pp. 226-251.

5. Nietzsche Fr., Also sprach Zarathustra. Ein Buch für Alle une Keinen (Ainsi parlait Zarathoustra. Un livre pour tous et pour personne, 1884), trad. Française de Geneviève Bianquis, $2 \mathrm{t}$., AubierFlammarion, Paris, 1969. Citation : $1^{\mathrm{e}}$ partie, t. I, pp. 170-171.

6. Andreev L., «Ben-Tovit », in : Nižegorodskij sbornik (Recueil de Nižnij-Novgorod), éd. Znanie, Saint-Pétersbourg, 1905, rééd. in : Sobr. soč., op. cit., pp. 555-558. Dans la traduction donnée en annexe, les passages en bleu correspondent aux extraits du texte cités dans le corps de l'étude.

7. Čekhov A., Čelovek v futljare (L'Homme à l'étui), in : Russkaja mysL', t. VII, 1898, pP. 120-131.

8. Cf. Andreev L., «V tëmnuju daL'» (Vers les lointains obscurs), in : Kur'er, Moscou, 25 décembre 1900, pp. 1-2.

9. Čekhov A., « Nevesta », in : Žurnal dlja vsekh, t. XII, 1903, colonnes 1413-1432.

10. Andreev L., Cari Iudejskie (Les Tsars de Judée, 1918). Il ne reste de ce tableau qu'une photo, reproduite (hors-texte) in : Andreev L., S.O.S., première édition du journal (1914-1919), des lettres, articles et entretiens de Leonid Andreev des années 1918 et 1919, sous la direction de Davies R. \& Hellman B., Atheneum-Feniks, Moscou-Saint-Pétersbourg, 1994.

11. Veresaev (pseud. de Smidovič) V., «Na estrade » (Sur le podium, 1900), in : Sobr. soč., t. I, Éditions de la société F. Marks, Saint-Pétersbourg, 1913, pp. 161-162.

12. Cf. Telešov N., Zapiski pisatelja (Carnets d'un écrivain), 1943 ; rééd.: Moskovskij rabočij, Moscou, 1966, p. 49.

13. Sur la notion de " petit-bourgeois ", cf. Rolet S., «La quête du bonheur et la figure du meščanin dans la Russie des derniers Romanov", in: Modernités russes, t. V, Lyon, 2004, pp. 113-122, rééd. In : Rolet S., Léonide Andréiev. L'Angoisse à l'œeuvre, préface de Lanne J.-Cl., Centre d'études slaves André Lirondelle, Université Jean Moulin-Lyon 3, Lyon, 2010, pp. 53-65.

14. Pour un aperçu du climat de l'époque, cf. Cinnella E., «La société russe à la veille de la révolution de 1905 ", in: 1905, la première révolution russe, sous la direction de Coquin $\mathrm{Fr}$-X. \& Gervais-Francelle C., Publications de la Sorbonne, Institut d'études slaves, Paris, 1986, pp. 539-560.

15. Lettre de Maksim Gor'kij à Leonid Andreev du 2 décembre 1901, in : Gor'kij M., Polnoe sobr. soč., Pis'ma $v$ dvadcati četyrëkh tomakh (Euvres complètes. Lettres en vingt-quatre volumes), t. II, Nauka, Moscou, 1997, p. 218.

16. Lettre de Maksim Gor'kij à Leonid Andreev du 23 janvier 1902, in : Gor'kij M., Poln. sobr. soč,, Pis'ma..., Ibid., t. III, p. 26. Maksim Gor'kij va jusqu'à écrire en 1905, quand la révolution a enfin éclaté : « Le premier jour de la révolution russe a été celui de la faillite morale [moraL’nogo krakha] 
de l'intelligentsia russe.» (Lettre de Maksim Gor'kij à Ekaterina Peškova, 9 janvier 1905, in : Perepiska M. Gor'kogo $v$ dvukh tomakh [Correspondance de M. Gor'kij en deux volumes], vol.1, Hudožestvennaja literatura, Moscou, 1986, p. 275).

17. Lettre de Maksim Gor'kij à Leonid Andreev du 2 décembre 1901, p. 217. Voir n. 15.

18. Idem.

19. Nietzsche Fr., Ainsi parlait Zarathoustra, op.cit., pp. 21-23 (l'édition est bilingue, seules les références de la traduction sont données ici [NdlA]).

20. Ibid, p. 23.

21. Idem.

22. Ibid., p. 27.

INDEX

Index chronologique : XXe siècle

Index géographique : Russie

Mots-clés : littérature russe, philosophie

oeuvretraite Andreev L. : Ben-Tovit

\section{AUTEURS}

\section{SERGE ROLET}

Professeur des universités, Université de Lille 3 (France), Centre de Recherche CECILLE 